\title{
Pravastatin was not better than usual care in reducing all cause mortality or CHD events
}

\author{
The Antihypertensive and Lipid-Lowering Treatment to Prevent Heart Attack Trial (ALLHAT-LLT). Major outcomes in \\ moderately hypercholesterolemic, hypertensive patients randomized to pravastatin vs usual care. JAMA \\ 2002;288:2998-3007. \\ QUESTION: In older patients with well controlled hypertension and moderately elevated \\ low density lipoprotein cholesterol (LDL-C), is pravastatin better than usual care in \\ reducing all cause mortality and coronary heart disease $(\mathrm{CHD})$ events?
}

\section{Design}

Randomised (allocation concealed*), unblinded,* controlled trial with mean 4.8 years of follow up (Antihypertensive and Lipid-Lowering Treatment to Prevent Heart Attack Trial [ALLHAT-LLT]).

\section{Setting}

513 clinical centres in the US, Canada, Puerto Rico, and the US Virgin Islands.

\section{Patients}

10355 patients (mean age $66 \mathrm{y}, 51 \%$ men) who were enrolled in the ALLHAT (age $\geq 55$ y and stage 1 or 2 hypertension with $\geq 1$ additional risk factor for CHD; fasting LDL-C concentration 3.1-4.9 mmol// for those with no known CHD, or $2.6-3.3 \mathrm{mmol} / 1$ for those with known $\mathrm{CHD}$; and fasting triglyceride concentrations $<3.9 \mathrm{mmol} / \mathrm{L}$ ). Patients were excluded if they were receiving lipid lowering treatment, large doses of niacin, or probucol; were intolerant of statins; or had liver or kidney disease, other contraindications for statin treatment, or a known secondary cause of hyperlipidemia. Follow up was 97\%. All randomised patients were included in the analysis.

\section{Intervention}

Patients were allocated to open label pravastatin, 40 $\mathrm{mg} /$ day $(\mathrm{n}=5170)$, or usual care (LDL-C lowering at the discretion of the primary care physician) $(\mathrm{n}=5185)$. $B$ R Davis, University

of Texas-Houston

Health Science Center,

Houston, TX, USA.

bdavis@sph.uth.tmc.edu

Abstract and

commentary also

appear in ACP Journal

Club

\section{Main outcome measures}

All cause mortality. Secondary outcomes included a composite of fatal CHD or nonfatal myocardial infarction (MI) (CHD events), cause specific mortality, and total and site specific cases of cancer.

Pravastatin v usual care for well controlled hypertension and moderately elevated low density lipoprotein cholesterol at 6 years

\begin{tabular}{lccll} 
Outcomes & Pravastatin & Usual care & RRR (95\% Cl) & NNT \\
All cause mortality & $14.9 \%$ & $15.3 \%$ & $1 \%(-11$ to 11$)$ & Not significant \\
\hline CHD events & $9.3 \%$ & $10.4 \%$ & $9 \%(-4$ to 21$)$ & Not significant \\
\hline
\end{tabular}

$\mathrm{TCHD}$ events $=$ a composite of fatal coronary heart disease or nonfatal myocardial infarction. Other abbreviations defined in glossary.
Main results

Analysis was by intention to treat. Pravastatin and usual care groups did not differ for all cause mortality (table). Groups also did not differ for CHD events (table) or for any other secondary outcomes.

\section{Conclusion}

In older patients with well controlled hypertension and moderately elevated low density lipoprotein cholesterol, pravastatin was no better than usual care in reducing all cause mortality and CHD events.

*See glossary.

\section{COMMENTARY}

Unlike the ALLHAT hypertension treatment trial, in which negative results justified a firm endorsement of a drug of first choice, the negative results of the companion ALLHAT lipid lowering trial (ALLHAT-LLT) are unimportant.

The negative results probably reflect flaws in study design and thus do not challenge beliefs about statins, especially in light of the positive results from the larger and more rigorous Heart Protection Study (HPS). ${ }^{1}$ The design flaw that sets it apart from the 9 other large, long term statin trials, including HPS, is the open label, unblinded design that allowed nearly $30 \%$ of the control group to "drop in" on lipid lowering treatment. By year 6 , the absolute difference in the reduction in LDL-C between the intervention group (28\%) and control group (16\%) was only $12 \%$.

At the start of ALLHAT-LLT in 1994, the value of statins for primary prevention among patients with only moderately elevated cholesterol and other cardiovascular risk factors was uncertain. Subsequent publication of several positive, blinded, placebo controlled, randomised trials provided convincing supporting evidence. Thus, only a meticulously designed, large, negative trial with minimal crossover could have challenged this conclusion. We agree with the authors' concluding advice to ignore the results and continue prescribing statins for patients with elevated risk for cardiovascular disease.

Arthur T Evans, MD, MPH Brian P Lucas, MD Cook County Hospital and Rush Medical College Chicago, Illinois, USA

1 MRC/BHF Heart Protection Study of cholesterol lowering with simvastatin in 20,536 high-risk individuals: a ran-
domised placebo-controlled trial. Lancet 2002;360:7-22. 